\title{
Alterations in early cytokine-mediated immune responses to Plasmodium falciparum infection in Tanzanian children with mineral element deficiencies: a cross-sectional survey
}

\author{
Erasto V Mbugi 1,7, Marjolein Meijerink1,2, Jacobien Veenemans ${ }^{1}$, Prescilla V Jeurink1,3, Matthew McCall4, \\ Raimos M Olomi5, John F Shao5, Hans Verhoef1,6 and Huub FJ Savelkou**1
}

\begin{abstract}
Background: Deficiencies in vitamins and mineral elements are important causes of morbidity in developing countries, possibly because they lead to defective immune responses to infection. The aim of the study was to assess the effects of mineral element deficiencies on early innate cytokine responses to Plasmodium falciparum malaria.

Methods: Peripheral blood mononuclear cells from 304 Tanzanian children aged 6-72 months were stimulated with $P$. falciparum-parasitized erythrocytes obtained from in vitro cultures.

Results: The results showed a significant increase by $74 \%$ in geometric mean of TNF production in malaria-infected individuals with zinc deficiency (11\% to 240\%; 95\% Cl). Iron deficiency anaemia was associated with increased TNF production in infected individuals and overall with increased IL-10 production, while magnesium deficiency induced increased production of IL-10 by $46 \%$ (13\% to 144\%) in uninfected donors. All donors showed a response towards IL-1 $\beta$ production, drawing special attention for its possible protective role in early innate immune responses to malaria.

Conclusions: In view of these results, the findings show plasticity in cytokine profiles of mononuclear cells reacting to malaria infection under conditions of different micronutrient deficiencies. These findings lay the foundations for future inclusion of a combination of precisely selected set of micronutrients rather than single nutrients as part of malaria vaccine intervention programmes in endemic countries.
\end{abstract}

\section{Background}

In African populations, multiple micronutrient deficiencies, infections and immunodeficiencies commonly coexist. Deficiencies in vitamins and mineral elements can impair immune responses to infectious diseases through multiple mechanisms, ranging from phagocytosis and innate immune responses to antibody formation and cellmediated immunity. Zinc is an important micronutrient because it is essential for the development, differentiation and function of several critical types of immune cells $[1,2]$. In vitro mitogen stimulation experiments indicate that marginal zinc deficiency can cause reduced counts of

\footnotetext{
* Correspondence: huub.savelkoul@wur.nl

1 Cell Biology and Immunology Group, Wageningen University, The Netherlands

Full list of author information is available at the end of the article
}

circulating leucocytes and reduced whole blood concentrations of cytokines, particularly IL-6 [3]. Zinc deficiency contributes to pneumonia, acute and chronic diarrhoea [4,5], and possibly malaria [4-6], which together constitute the leading causes of death in African children. In addition, zinc deficiency may exacerbate the outcome of diseases such as HIV and tuberculosis that rely on macrophage killing of infected cells [7]. Deficiencies of copper [8], iron and vitamin $B_{12}$ have been associated with impaired neutrophil functions whereas deficiencies of folic acid are not [9].

A fast-acting innate immune response, mediated by cytokines such as interleukine-1 $\beta$ (IL-1 $\beta$ ), IL-12 and tumour necrosis factor (TNF), is crucial for host survival in the initial stages of Plasmodium falciparum infection [10]. Zinc is needed for monocytes and macrophages to 
produce IL-1 $\beta$ and for other peripheral blood mononuclear cells (PBMCs) to produce TNF- $\alpha$. Zinc deficiency can lead to impaired phagocytosis and intracellular killing by macrophages and neutrophils. In addition, it can impair NK-cell function, cytokine production, the generation of an oxidative burst as well as complement activity [2,11-14] through decreased activation of various cellular responses and low concentrations of IL- $1 \beta$. In addition, innate immune responses determine the type and efficiency of subsequent adaptive immune responses $[10,15,16]$ at later stages of infection.

This study was conducted to assess the impact of deficiencies of zinc and other mineral elements on early innate immune responses to $P$. falciparum infection. This micronutrient deficiency was assessed in vitro by stimulation experiments, using PBMCs samples that were collected from Tanzanian children aged 6-72 months. The assumptions were that zinc deficiency alters the balance in cytokine production and their association in early immune responses, and that deficiencies of zinc and other mineral elements induce a decreased ability of PBMCs to produce pro-inflammatory cytokines, and the regulatory cytokine IL-10, when exposed to P. falciparum parasites. In addition, it was analysed to what extent the magnitude of the PBMCs cytokine response depended on the P. falciparum infection status of the child at the time that the blood was collected and PBMCs were isolated.

\section{Methods}

\section{Study area and population}

This study was conducted in a lowland area around Segera village (S $05^{\circ} 19.447^{\prime}$, E $\left.38^{\circ} 33.249^{\prime}\right)$, Handeni District, north-eastern Tanzania, in May-July 2006. Malaria is highly endemic in this area, with virtually all infections being due to $P$. falciparum. The residents in the study population mostly comprise poor farmer families growing maize and cassava for subsistence use. Such populations are prone to deficiencies of zinc and iron because they have cereal-based diets that are rich in natural dietary constituents that inhibit the absorption of these trace metals [17]. At the time of our study, only one health centre in Segera was available to serve all of the surrounding area. The study was approved by Ethics Review Committees in The Netherlands and Tanzania (reference numbers for KCMC and the National Health Research Ethics Review sub-Committee: 094 and NIMR/ $\mathrm{HQ} /$ R.8a/VolIX/540, respectively). Informed consent was obtained from community leaders and local government officials, and from parents or guardians.

\section{Sampling methods and eligibility criteria}

A census list was made with all resident children aged 672 months in the study area. Using this list, 16 children were randomly selected from 19 communities, resulting in a total of 304 subjects. Further details are provided elsewhere [18].

\section{Field procedures}

All children were examined by a clinical officer, who also measured axillary temperature by electronic thermometer. Subjects were eligible when they had no fever, and showed no signs of other severe disease or severe malnutrition (weight-for-height z-score below -3 SD). Plasmodium infection was detected both by microscopy and rapid immunochromatographic assay (Vista Diagnostics Int. Kirkland, WA, USA, based on antibodies developed for OptiMAL test by Flow Inc., Portland, OR, USA). Although this dipstick test cannot be used to determine the duration of infection, it detects lactate dehydrogenase (pLDH) produced by live parasites only, either $P$ falciparum or any human Plasmodium species $[19,20]$. For blood samples with > 50 P. falciparum parasites $/ \mathrm{mL}$ (0.001\% parasitaemia), it has been found that the OptiMAL assay has a sensitivity of approximately $96 \%$ [20]. Venous blood $(6 \mathrm{~mL})$ was collected in containers suitable for mineral element analysis with sodium heparin as anticoagulant (Becton-Dickinson, Franklin Lakes, NJ). Immediately upon collection, the cap was sprayed with ethanol and allowed to dry; approximately $1.3 \mathrm{~mL}$ blood was then drawn by sterile syringe. This aliquot was centrifuged and plasma samples were stored and transported to The Netherlands at $-80^{\circ} \mathrm{C}$ for subsequent measurement of mineral element concentrations. The remainder of the blood sample was kept at $20-25^{\circ} \mathrm{C}$ during transport the same day to the laboratory in Moshi, at approximately $300 \mathrm{~km}$ distance, for collection of additional plasma and PBMCs. Children were treated for common childhood infections and anaemia according to guidelines of Tanzanian Ministry of Health.

\section{Determination of plasma concentrations of mineral elements}

Plasma samples were diluted 20 times in milliQ [21], and concentrations of zinc, magnesium and copper were measured by inductively-coupled plasma atomic emission spectrometry (ICP-AES) (Vista Axial, Varian, Australia). To determine variability in outcomes, measurements were replicated five times. With mean values set at $100 \%$, measurements varied between $97 \%$ to $102 \%$ for zinc, $99 \%$ to $102 \%$ for magnesium, and $97 \%$ and $102 \%$ for copper. Because we found no evidence for copper deficiency as assessed by plasma copper concentrations $<7.1 \mu \mathrm{mol} / \mathrm{L}$ (unpublished data), only the results for zinc and magnesium are reported in this paper.

\section{Determination of plasma indicators of iron stores and inflammation}

After arrival at the laboratory in Moshi, blood samples were immediately centrifuged $(300 \times g)$ at ambient tem- 
peratures for 10 minutes. Plasma $(1.2 \mathrm{~mL})$ was collected and replaced this immediately with an equal volume of Iscove's modified Dulbecco's medium (IMDM) with GlutaMAX (Invitrogen Gibco-BRL, Life Technologies, Grand Island, NY, USA) for subsequent isolation of PBMCs (see below). Plasma was stored in liquid nitrogen, and subsequently transported on dry ice to The Netherlands, where plasma concentrations of ferritin and $\mathrm{C}$-reactive protein were measured as indicators of iron stores and inflammation, respectively, by using a Behring nephelometer (BN ProSpec; Dade-Behring) in The Netherlands (by dr. J. P. M. Wielders at the Meander Medical Centre, Amersfoort, the Netherlands).

\section{PBMCs isolation}

PBMCs were isolated by Ficoll density gradient centrifugation, cells were transferred to $10 \% \mathrm{v} / \mathrm{v}$ DMSO in fetal calf serum, cooled at $-1^{\circ} \mathrm{C} /$ minute in an isopropyl-loaded device (Nalgene, Rochester, NY, USA) and preserved in liquid nitrogen [22]. For a 16-h period during transport to Wageningen University, The Netherlands, the PBMCs were kept on dry ice, and immediately thereafter stored again in liquid nitrogen until stimulation experiments.

\section{Preparation of P. falciparum-parasitized and unparasitized erythrocytes}

Routinely prepared asexual stage parasitized erythrocytes were obtained from the Department of Medical Microbiology, Radboud University, Nijmegen [23]. Briefly, human $\mathrm{O}$ and rhesus-negative erythrocytes from healthy blood donors (Sanquin, Nijmegen, The Netherlands) were cultured in medium to which live $P$. falciparum parasites (NF54 strain) produced in a continuous culture were added $[23,24]$. After two to four days, when $\sim 8-10 \%$ of erythrocytes were parasitized by asexual Plasmodium stages, the culture was concentrated by centrifugation at $625 \times g$ for $5 \mathrm{~min}$; parasitized erythrocytes were separated on a 67\% Percoll gradient as reported elsewhere [25] and washed twice in phosphate-buffered saline (PBS). Purified parasitized erythrocytes were preserved at a concentration of approximately $15 \times 10^{7} / \mathrm{mL}$ in $13 \%$ glycerol $/ \mathrm{PBS}$ in a freezing container at $-80^{\circ} \mathrm{C}$. Glycerol $(50 \% \mathrm{w} / \mathrm{v})$ was added to the parasitized erythrocytes to avoid mechanical damage of the cells through ice formation. Unparasitized erythrocytes were processed similarly but without adding parasites to serve as a control. Both in parasitized and unparasitized erythrocyte cultures, we confirmed the absence of mycoplasma contamination by polymerase chain reaction. Both parasitized and unparasitized erythrocytes were counted by flow cytometry, and compared regarding their size and internal complexity to the counting beads and PBMCs. Aliquots were made and stored at $-8^{\circ} \mathrm{C}$ until needed for PBMCs stimulation.

\section{PBMCs stimulation}

Malaria antigens differ in their capabilities to stimulate PBMCs: intact parasitized red blood cells ( $\mathrm{pRBC}$ ) are capable of inducing more rapid and intense pro-inflammatory responses from PBMCs than freeze-thaw lysates of $P$. falciparum [26]. To simulate in vivo malaria-specific responses as closely as possible, $P$. falciparum $\mathrm{pRBC}$ were used, with an adapted protocol for stimulation of PBMCs by Jeurink et al [22]. In brief, PBMCs were cultured at $10^{6}$ cells/well in sterile polystyrene 48-well plates with flatbottom wells (Corning Inc, Corning, NY, USA). Based on initial optimization experiments, aliquots of $\mathrm{pRBC}$ were thawed and cultured with PBMCs in IMDM with glutamax containing Yssel's supplements [27] with 2\% human $\mathrm{AB}$ serum, $1 \%$ penicillin/streptomycin and $1 \%$ fungizone (Gibco-BRL), at a PBMCs:pRBC ratio of 1:2. PBMCs were also cultured under similar conditions with unparasitized erythrocytes $(\mathrm{uRBC})\left(2 \times 10^{6}\right.$ cells/well $)$ as a negative control, and with soluble antibodies to CD3 and soluble antibodies to CD28 (Cat. No.555336 and 555725, BectonDickinson, Alphen aan den Rijn, The Netherlands) as a positive control. Monoclonal anti-CD3 and anti-CD28 antibodies provide co-stimulatory signals and polyclonal stimulation required for maximal proliferation of $\mathrm{T}$ lymphocytes [22,28]. Cell culture plates were incubated at $37^{\circ} \mathrm{C}$ in a humidified atmosphere containing $5 \% \mathrm{CO}_{2}$. After PBMCs culturing for 1 day, we aspirated $75 \mu \mathrm{L}$ of the supernatant per well to measure cytokine concentrations.

\section{Measurement of cytokine concentrations}

Concentrations of IL-1 $\beta$, IL-10, IL-12p70 and TNF were determined on a FACSCanto II flow cytometer by cytometric bead array system and analysed with FCAP software (all from Becton-Dickinson).

\section{Statistical analysis}

Data were entered and analysed using SPSS for Windows (version 15.0. SPSS Inc., Chicago, IL, USA). Zinc deficiency and low zinc status were defined as plasma zinc concentrations $<9.9 \mu \mathrm{mol} / \mathrm{L}$ and $<10.7 \mu \mathrm{mol} / \mathrm{L}$, respectively. Low magnesium status was defined by magnesium concentration $<750 \mu \mathrm{mol} / \mathrm{L}[29,30]$. Iron deficiency anaemia was defined by co-existing iron deficiency (plasma ferritin concentration $<12 \mu \mathrm{g} / \mathrm{L}$ ) and anaemia (haemoglobin concentration $<110 \mathrm{~g} / \mathrm{L}$ ). Fisher's Exact Test was performed to determine the association between inflammation (CRP levels) and sex, age, malaria status as well as nutritional status. Cytokine concentrations were ln-transformed to obtain normally-distributed values. Group differences in these values were analysed assuming $\mathrm{t}$-distributions. Interactions between malaria and micronutrient indicators were assessed using multi- 
ple linear regression models on log-transformed cytokine data; the resulting effect sizes were exponentiated and expressed as percentage values. Linear regression analyses were also carried out to explore the associations between IL-1 $\beta$, TNF and IL-10, and to what extent these associations were influenced by nutrient status and malaria infection status. The association between concentrations of TNF and IL-10 were considered as a measure of balance between the pro-inflammatory responses and the regulatory response. The analyses of the cytokine responses to $\mathrm{pRBC}$ are reported. As expected, the average response to $\mathrm{URBC}$ (negative control) was less than to pRBC, whereas the average response to CD3/CD28 (positive control) was higher. Correction for these responses does not change the estimates of the associations between nutrient status and cytokine responses, or between malarial infection status and cytokine responses.

\section{Results}

\section{General characteristics of the study population}

Peripheral blood was collected from 135 boys and 169 girls; these had similar age distributions. The following prevalence values (n) were found: low zinc status: $63.1 \%$ (188); zinc deficiency: $48.3 \%$ (144); low magnesium status: $65.1 \%$ (194); iron deficiency anaemia: 9.4\% (26); malaria: $46.1 \%$ (140). Malaria and age were associated with inflammation (determines as CRP levels); however, there was no evidence that inflammation was associated with zinc deficiency, magnesium deficiency or iron deficiency anaemia (Fisher's Exact Test). Detailed characteristics of the study population by malarial infection status are summarised in Table 1 . In addition, the associations between nutrient status and supernatant cytokine concentrations, and between malaria infection status of the child at the time of blood collection and supernatant cytokine concentrations, following $24 \mathrm{~h}$ of PBMCs stimulation with $P$. falciparum-infected erythrocytes are also summarized (Figure 1). Adjustment for age class, sex and/or magnesium deficiency did not lead to marked changes in the associations between zinc deficiency and supernatant cytokine concentrations shown in Figure 1; conversely, adjustment for age class, sex and/or zinc deficiency did not lead to marked changes in the associations between magnesium deficiency and those supernatant cytokine concentrations.

\section{Association between nutrient indicators and in vitro innate cytokine production, by malaria infection status at the time of blood collection}

When analysing IL-10 concentrations, all individuals with IL-10 concentrations below the detection limit were excluded. In some instances, differences in cytokine concentrations between nutrient replete and deficient children (Figure 2) seemed to depend on malarial infection status at the time of blood collection (Table 2). The profile of supernatant cytokine concentration appeared different between subjects with deficiencies in zinc, magnesium and with iron deficiency anaemia. In the absence of malaria infection at the time of blood collection, zinc deficiency was associated with marginal reductions in concentrations of TNF, IL-1 $\beta$ and IL-10. Amongst donors with malaria infection at the time of blood collection, zinc status was not associated with altered concentrations of IL- $1 \beta$ or IL-10, but low plasma zinc concentrations were associated with an increase in TNF concentration by $74 \%$ ( $11 \%$ to $240 \%, 95 \% \mathrm{CI})$. Malaria infection at the time of blood collection seemed to determine the magnitude of the association between low plasma zinc concentration and TNF concentration (9\% reduction in children without malaria, as compared to $74 \%$ increase in their peers with malaria; although the statistical evidence for this difference was weak $(P=0.15)$.

Magnesium deficiency, on the other hand, was associated with increased concentrations of IL-10; this increase was $46 \%$ in children without malaria, as compared to only $6 \%$ in their peers with malaria (Figure 2). Low magnesium concentrations seemed associated with reduced concentrations of TNF and IL- $1 \beta$ by $-25 \%$ (95\% CI: $-64 \%$ to $55 \%$; $P=0.79)$ and $-44 \%(-70 \%$ to $6 \% ; P=0.13)$, respectively, in children with malaria infection at the time of blood collection, although these differences may have been due to chance. These results are a reverse of the situation in zinc deficiency. The numbers of individuals with both iron deficiency anaemia and malaria (Table 1) were too low to compare groups meaningfully.

\section{Influence of malaria and nutrient indicators on associations between cytokine concentrations}

No evidence was found that the associations between concentrations of TNF and IL-10 depended on zinc, magnesium or malaria status at time of blood collection, as indicated by differences in slopes of $17 \%(-44 \%$ to $147 \%$; $95 \% \mathrm{CI}, P=0.67)$ for zinc status, $10 \%(-47 \%$ to $127 \%$; $95 \%$ CI, $P=0.80)$ for magnesium status, or $3 \%(-24 \%$ to $39 \%$; $95 \% \mathrm{CI}, P=84$ ) for malaria (Figure 3 ). There was a tendency, albeit not significant, that iron deficiency anaemia (IDA) influenced the relationship between concentrations of TNF and IL-10, as indicated by the difference between slopes of $119 \%$ (35\% to 637\%; $95 \%$ CI, $P=0.20$ ).

Additional linear regression analyses (Figure 4) showed evidence that zinc status influenced the association between concentrations of IL-1 $\beta$ and IL-10, as indicated by differences in slopes of $118 \%$ (4\% to $359 \%$; $95 \%$ CI, $P=$ $0.04)$. There was no evidence that malaria infection influenced the association between concentrations of TNF and IL-1 $\beta$ (Figure 4, malaria panel), as indicated by a difference in the slopes of regression lines of $9 \%(-13 \%$ to $35 \% ; P=0.47)$. In summary, these results show no evi- 

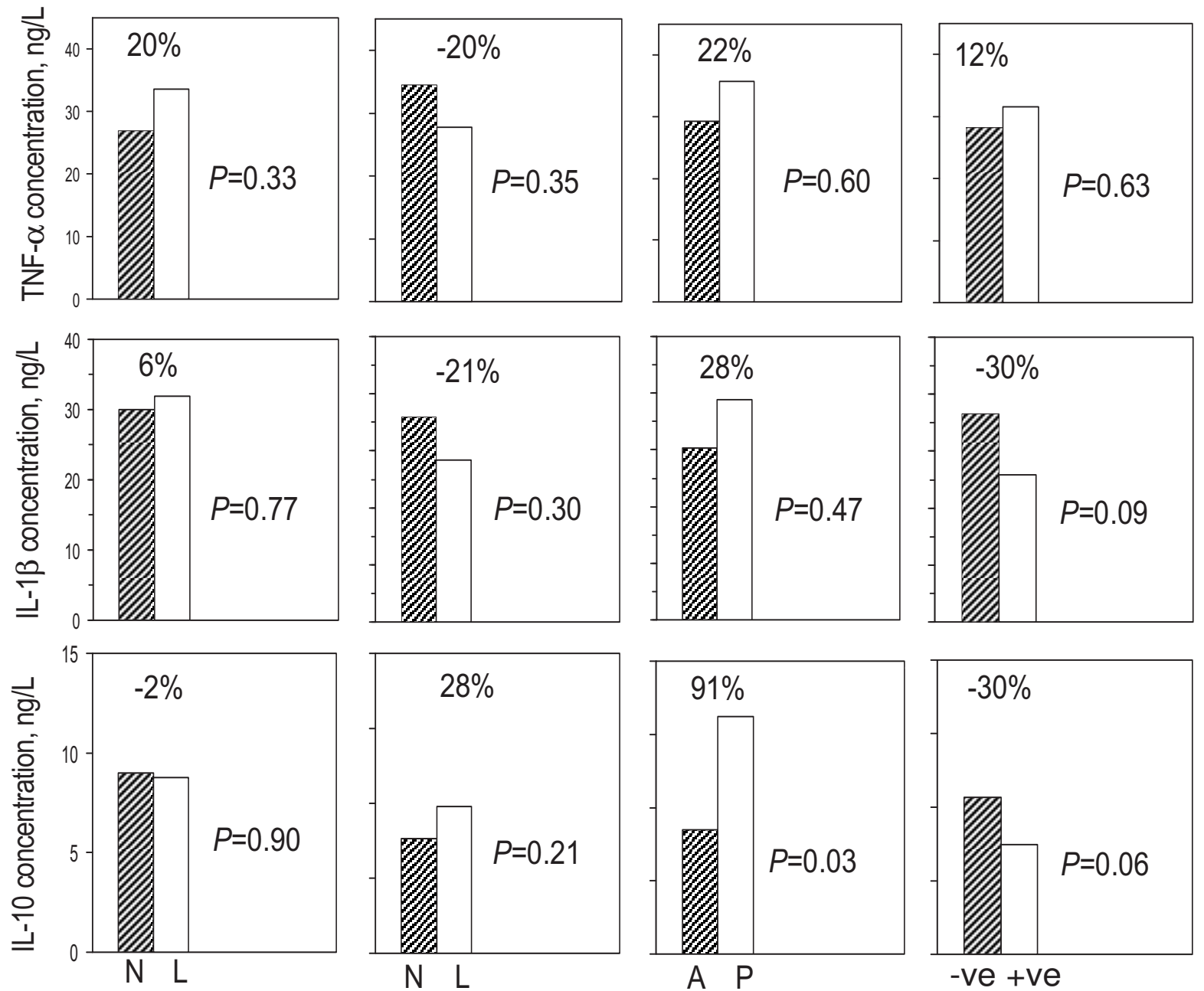

\section{Zinc concentration}

Magnesium conc.

Iron deficiency anaemia

Malaria

Figure 1 Associations between nutrient status and supernatant cytokine concentrations, and between malaria infection status of the child at the time of blood collection and supernatant cytokine concentrations, following $24 \mathrm{~h}$ of PBMC stimulation with Plasmodium falciparuminfected erythrocytes. N: normal concentrations; L: low concentrations (plasma concentrations of zinc and magnesium $<9.9 \mu \mathrm{mol} / \mathrm{L}$ and $<750$ $\mu \mathrm{mol} / \mathrm{L}$, respectively); A: absence of iron deficiency anaemia; P: presence of iron deficiency anaemia (co-existing iron deficiency; plasma ferritin concentration $<12 \mu \mathrm{g} / \mathrm{L}$ and anaemia; haemoglobin concentration $<110 \mathrm{~g} / \mathrm{L}$ ). -ve: malaria negative; +ve: malaria positive. Percentages indicate paired group differences in supernatant cytokine concentrations, expressed as percentages relative to values observed in groups with normal plasma zinc or magnesium concentrations. P-values were obtained by assessing by multivariate analysis to what extent the proportional change in cytokine concentration that is associated with nutrient or malaria status.

dence of influence on associations among innate cytokines, by the various conditions of micronutrient and malaria status at time of blood collection except for zinc status, for which there was some evidence that it influenced the association between IL-1 $\beta$ and IL-10. There was no evidence of an influence of micronutrient status and malaria on associations in other relationships. There were insufficient cases in all groups to explore and meaningfully compare the associations between IL-12 and other cytokines.

\section{Discussion}

Effects of plasma concentrations of mineral elements on in vitro cytokine responses by PBMCs

The biochemical data showed that most children in this study had nutrient deficiencies, particularly in zinc and magnesium and to a lesser extent iron deficiency anaemia. Zinc deficiency was associated with increased TNF responses in children with malaria infection at the time of blood collection but not in those without infection. TNF is a pro-inflammatory cytokine resulting in pathology if 
Table 1: Characteristics of the study population, by malarial infection status

\begin{tabular}{|c|c|c|c|}
\hline & Plasmodium-infected & Plasmodium-uninfected & P-value \\
\hline Sex & & & 0.56 \\
\hline Male & 65 & 70 & \\
\hline Female & 75 & 94 & \\
\hline Age class & & & 0.03 \\
\hline 6-12 months & 7 & 19 & \\
\hline $12-24$ months & 18 & 31 & \\
\hline 24-48 months & 61 & 58 & \\
\hline 48-72 months & 54 & 55 & \\
\hline Zinc deficient ${ }^{1}$ & & & 0.49 \\
\hline Yes & 63 & 81 & \\
\hline No & 74 & 80 & \\
\hline Magnesium deficient ${ }^{2}$ & & & 0.63 \\
\hline Yes & 87 & 107 & \\
\hline No & 50 & 54 & \\
\hline Iron deficiency anaemia 3 & & & $<0.001$ \\
\hline Yes & 2 & 24 & \\
\hline No & 138 & 140 & \\
\hline
\end{tabular}

not properly regulated. In children with malarial infection, zinc deficiency was associated with increased production of IL-1 $\beta$ and IL-10, even if this increase did not bring the levels to those reached by individuals in the non-infected group. This is important because IL-10 is required to limit the production of pro-inflammatory cytokines, so that they do not lead to pathological consequences [31]. The low production of IL-10, however, could be due to the fact that the cytokine is said to be produced late (in vivo) following infection relatively to the innate cytokines. The initial production of TNF could also be the triggering factor by feedback mechanisms for production of IL-10 although Ramharter et al [32] reported increased responsiveness of in vivo primed cells as compared to malaria-naïve cells, with a tendency towards increased production of TNF. This can possibly explain the difference between subjects who were exposed or non-exposed at the time of blood collection, in response to in vitro stimulation in our study. These results show possible alterations in innate cytokine production particularly TNF and IL1- $\beta$ due to the reported impaired macrophage functions and NK-cells activity in zinc deficiency $[1,2,13,33]$. Interaction between these cells leads to the production of innate cytokines in the early stages of infections.

The relatively higher cytokines levels in individuals with malarial infection as compared to their uninfected peers (Figure 2), however, can be explained by the priming of the immune system by malaria. Exposure of T cells to a plethora of Plasmodium antigens leads to priming, so that these cells during subsequent exposures even with subsets of these antigens can produce greatly increased amounts of IFN- $\gamma$. This cytokine is necessary for up-regulation of production of TNF and other pro-inflammatory cytokines by monocytes, but also Th subsets and even natural killer cells, in malaria infection [26,34]. The cellular source of these abundantly produced cytokines, like IL-1 $\beta$ and IL-10 remain to be established in future studies, as besides monocytes/macrophages and B-cells, also various T-cell subsets will be involved. The increase in innate cytokine production in zinc-deficient individuals with malarial infection can be the result of a shift in activated monocytes towards a pro-inflammatory immune response, associated increased levels of TNF and IL-1 $\beta$, due to zinc deficiency in combination with prior priming of these cells due to previous exposure to malaria, as has been suggested before [35]. The initial contact with the pathogen directs towards production of pro-inflammatory cytokines to limit infection. Loharungsikul et al [36] proposed Toll-like receptors (TLRs) to play a role in innate immune recognition in which the differential expression of TLRs on antigen presenting cells (APCs) could be regulated by the P. falciparum parasite. This could account for the increase in levels of TNF in 

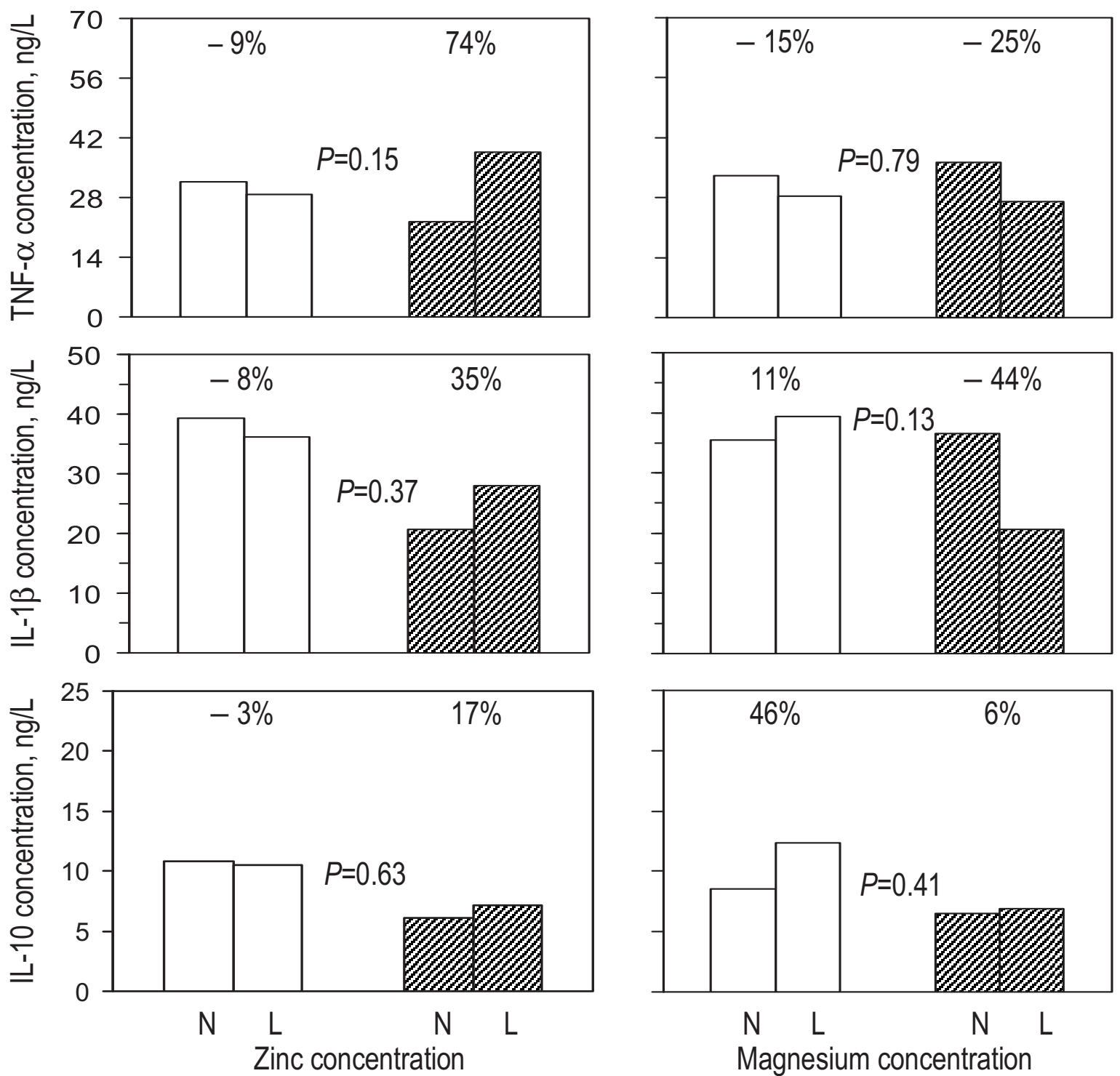

Figure 2 Associations between nutrient status and supernatant cytokine concentrations following $24 \mathrm{~h}$ of PBMC stimulation with Plasmodium falciparum-infected erythrocytes, by malaria infection status of the child at the time of blood collection. N: Normal concentrations; L: low concentrations (plasma concentrations of zinc and magnesium $<9.9 \mu \mathrm{mol} / \mathrm{L}$ and $<750 \mu \mathrm{mol} / \mathrm{L}$, respectively). Data from children without and with malaria infection at the time of blood collection are indicated with open and shaded columns, respectively. Percentages indicate group differences in supernatant cytokine concentrations, expressed as percentages relative to values observed in groups with normal plasma zinc or magnesium concentrations. $P$-values were obtained by assessing by multivariate analysis to what extent the proportional change in cytokine concentration that is associated with nutrient status is different between children with and without malarial infection. The number of individuals with iron deficiency and malaria (table 1) was too small to meaningfully compare among groups.

malaria-positive individuals regardless of micronutrient status (Figure 1). Glycosylphosphatidyl inositols (GPIs) that anchor $P$. falciparum merozoite surface protein 1 (MSP1) and merozoite surface protein 2 (MSP2) were described to be the pathogen associated molecular patterns (PAMPs) preferentially recognised by TLR-2 and TLR-4 [37]. The recognition and the interaction between these molecular patterns signal the induction of proinflammatory cytokine production. In addition, it is possible that parasite DNA attached to malarial pigment (haemozoin) produced in the course of infection further activates the innate immune response through TLR-9 engagement [38]. The expression of TLRs has been found to differ between malaria-infected and uninfected indi- 
Table 2: Influence of nutritional indicators on innate cytokines responses after 24-h in vitro stimulation of PBMCs with malaria-infected erythrocytes

\begin{tabular}{|c|c|c|c|}
\hline \multirow[t]{2}{*}{ Nutrient status indicator } & \multicolumn{3}{|c|}{ Supernatant concentration (ng/L) after $24 \mathrm{~h}$ of stimulation } \\
\hline & TNF- $a$ & IL-1 $\beta$ & IL-10 \\
\hline \multicolumn{4}{|l|}{$\begin{array}{c}\text { Children without } \\
\text { Plasmodium infection }\end{array}$} \\
\hline \multicolumn{4}{|c|}{ Plasma zinc concentration } \\
\hline Normal & $31.7 \quad(32)$ & $39.3 \quad(37)$ & $10.8 \quad(45)$ \\
\hline Low & $28.8 \quad(28)$ & $36.2 \quad(36)$ & $10.5 \quad(20)$ \\
\hline Difference & $-9 \%(-51 \%$ to $67 \%)$ & $-81 \%(-49 \%$ to $68 \%)$ & $-3 \%(-42 \%$ to $63 \%)$ \\
\hline \multicolumn{4}{|l|}{$\begin{array}{l}\text { Plasma magnesium } \\
\text { concentration }\end{array}$} \\
\hline Normal & $33.2 \quad(25)$ & $35.5 \quad(30)$ & $8.5 \quad(18)$ \\
\hline Low & $28.4 \quad(35)$ & $39.4 \quad(43)$ & $12.4 \quad(27)$ \\
\hline Difference & $-15 \%(-54 \%$ to $59 \%)$ & $11 \%(-40 \%$ to $104 \%)$ & $46 \%(13 \%$ to $144 \%)$ \\
\hline \multicolumn{4}{|l|}{ Iron deficiency anaemia } \\
\hline Absent & $27.1 \quad(51)$ & $35.3 \quad(63)$ & $9.3 \quad(37)$ \\
\hline Present & $33.9 \quad(11)$ & $43.8 \quad(13)$ & $19.9 \quad(8)$ \\
\hline Difference & $25 \%(-45 \%$ to $183 \%)$ & $24 \%(-42 \%$ to $170 \%)$ & $113 \%$ (13\% to $301 \%)$ \\
\hline
\end{tabular}

Children with

Plasmodium infection

Plasma zinc concentration

Normal
Low
Difference

$22.2 \quad(27)$

$38.7 \quad(31)$

$74 \%$ (11\% to $240 \%)$

Plasma magnesium concentration

$\begin{array}{lrl}\text { Normal } & 36.3 & (19) \\ \text { Low } & 27.2 & (39) \\ \text { Difference } & -25 \% & (-64 \% \text { to } 55\end{array}$

Iron deficiency anaemia

$\begin{array}{lll}\text { Absent } & 31.6 & (61) \\ \text { Present } & 64.9 & (1)\end{array}$

$20.7 \quad(27)$

$35 \%$ ( $26 \%$ to $146 \%$ )
$36.5 \quad(19)$

$20.6 \quad(43)$

$-44 \%(-70 \%$ to $6 \%)$
$6.1 \quad(12)$

$7.2 \quad(18)$

$17 \%$ (-34\% to $107 \%)$

Difference Not calculated

$26.1 \quad(64)$

$17.7 \quad(2)$
$6.5 \quad(12)$

6.9 (18)

$6 \%(-40 \%$ to $87 \%)$ Not calculated

$-32 \%(-88 \%$ to $270 \%)$

7.6

$3.2 \quad(1)$

Values indicate geometric means ( $\mathrm{n}$ ) or effect [95\% Cl]. Cut-off values to define low plasma concentrations of nutritional indicators: see text. When computing differences between values, the category with normal plasma concentration was used as the reference. 'With malaria' and 'without malaria' refers to infection status of children studied at the time of blood collection.

viduals, with higher expression being observed in infected patients $[24,39]$. These recent studies have further indicated TLR-2 to be highly expressed in mononuclear cells, particularly monocytes of P. falciparuminfected children and that TLR-2 are well responsive following stimulation with $\mathrm{pRBCs}$ resulting into stronger signals with consequential change in cytokine production profiles.
Unfortunately, the design of this study did not allow to ascertain the duration of infection at the time of blood collection. The children investigated may have been infected for some time, and may not have relied on the innate cytokines measured to control infection at the time of blood collection. It is also important to note that this concerned a cross-sectional study, that samples that were collected in a single time point, and that cytokines 


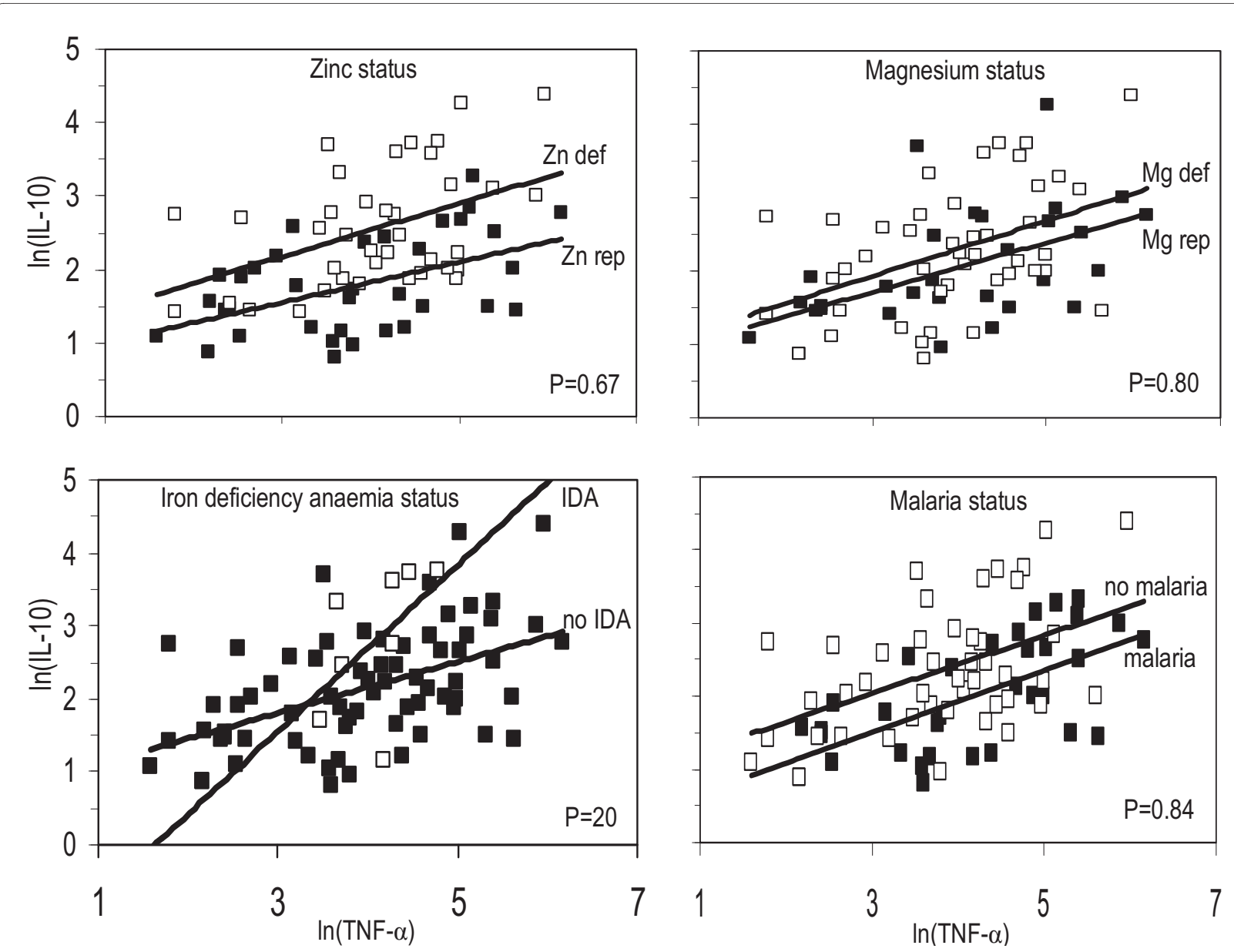

Figure 3 Associations between supernatant concentrations of TNF- and IL-10 following $24 \mathrm{~h}$ stimulation of peripheral blood mononuclear cells with Plasmodium falciparum-infected erythrocytes, by micronutrient and malaria status at the time of blood collection. Black blocks = zinc or magnesium replete, no iron deficiency anaemia or no malaria; open blocks = zinc and magnesium deficiency, iron deficiency anaemia or positive results for malaria tests at time of blood collection. $P$-values indicate probabilities of obtaining differences in associations between cytokine concentrations (as indicated by the slopes of the lines) as least as extreme as observed, assuming no differences.

measured had accumulated in supernatant following 24-h stimulation of cultured cells. The design of our study does not allow time-dependent production of cytokines, or to establish specific cell subsets are sources of the cytokines measured.

The interesting result in this study is that the impact of magnesium deficiency on early cytokine responses followed a different profile from that observed with zinc status. Magnesium deficiency seemed to be associated overall with low TNF concentrations, low concentrations of IL- $1 \beta$ and higher concentrations of IL-10 in uninfected but not infected donors (Figure 2). Low levels of proinflammatory cytokines in malaria are critical because they reduce the ability of the initial innate immune response to limit infection. These results imply that magnesium deficiency directs early cytokine responses towards anti-inflammatory rather than pro-inflammatory cytokine responses, although further studies are still needed to confirm this hypothesis. The significantly increased IL-10 and variable alteration in levels of TNF and IL-1 $\beta$ in both malaria-negative and malaria-positive subjects with magnesium deficiency may explain the imbalance in cytokine production as a result of magnesium deficiency modulated by malaria status.

Methodological differences may explain contradictions between our findings and those from previous studies $[7,9,40]$. Parasitized erythrocytes were used to simulate the in vivo infection, whereas others used mitogens, lipopolysaccharides (LPS), phytohemagglutinin (PHA) and polyclonal stimulation. In addition, we used Ficollisolated PBMCs that had been stored for several months under frozen conditions, whereas whole blood stimulated within 15 minutes of collection was also used in some of the previous studies. McCall et al [24] stimulated freshly 

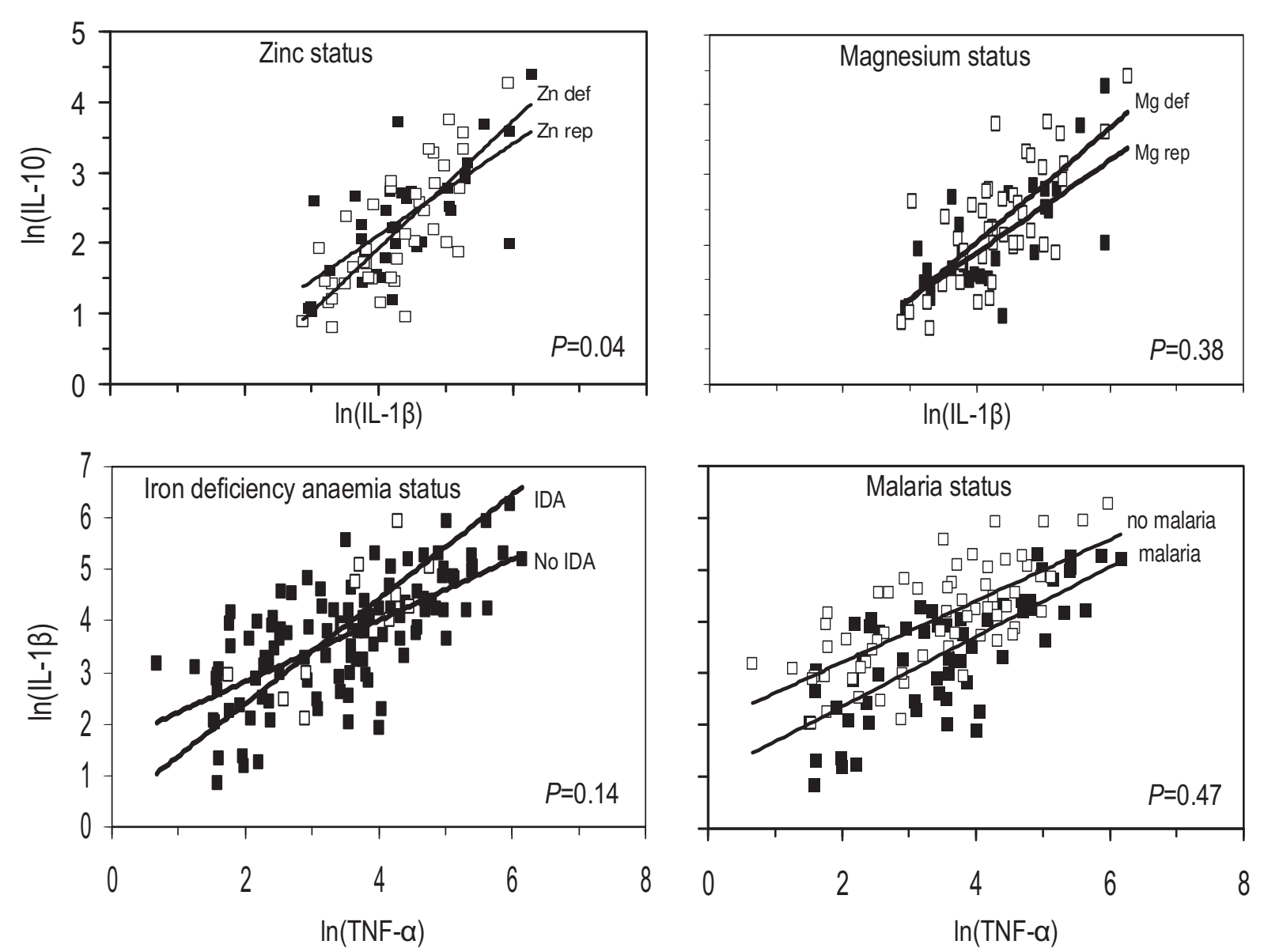

Figure 4 Relationships between supernatant concentrations of TNF- $\alpha$, IL-1 $\beta$ and IL-10 following $24 \mathrm{~h}$ stimulation of PBMCs with Plasmodium falciparum-infected erythrocytes, under different conditions of micronutrient and malaria status at the time of blood collection. Black blocks = zinc replete, magnesium replete, or no malaria; open blocks = zinc deficiency or positive results for malaria test at time of blood collection.

prepared PBMCs from adult naïve volunteers ex vivo with $P$. falciparum antigens. These findings suggest that zinc and other micronutrients can protect against malaria infection by a different means such as targeting specific pathogenic processes of infection in vivo [41]. Nevertheless, the idea that zinc can also reduce production of proinflammatory cytokines by inhibiting signal transduction in monocytes in healthy human subjects [42], particularly IL- $1 \beta$ and TNF $[2,43,44]$, should be further explored. The latter idea is also supported by in vitro studies $[45,46]$ in other conditions than malaria.

The results from this study and those conducted by others $[47,48]$, IL-12 concentrations were below the detection limits. The most probable reason is the time required for maximal priming of pathogen recognition receptors (e.g. TLRs) on PBMCs by P. falciparum-parasitized erythrocytes. McCall et al [24] have shown that proinflammatory priming effects of $P$. falciparum require up to 48 hours to develop maximally, whereas we measured cytokines after 24 hours of stimulation. This priming is lacking in our culture system despite the reported poor in vitro induction of IL-12 by P. falciparum [49]. IL-12 levels obtained in vitro from stimulated monocytes and macrophages are generally low and zinc deficiency is reported to be associated with further decreased IL-12 production [50]. Early IL-12 activity is also liable to suppression by transforming growth factor (TGF) $\beta[51,52]$ that has been reported to variably influence and result in weak IL-12 activation and production, at least in vivo. Most of our donors responded towards production of IL-1 $\beta$ rather than TNF and IL-10. This is interesting since although different arguments reveal the pathological effect of IL$1 \beta$ on cerebral malaria and severity of the disease in children [53], IL-1 $\beta$ together with other pro-inflammatory cytokines like IFN- $\gamma$ and IL- 6 is said to be protective against malaria by inducing parasite killing by monocytes, macrophages and neutrophils [54]. Production of $\mathrm{IL}-1 \beta$ is induced by direct interaction between zinc and 
monocytes through activation of interleukine-1 receptor associated kinase (IRAK) which is dose-dependent [44]. Lower in vivo zinc levels, partially inhibit IRAK leading to diminished but not completely inhibited normal T-cells IL- $\beta$ response. Results from this study may also reflect that stimulation of cryopreserved PBMCs by pRBCs results in a gradual production of innate cytokines preceded by IL- $1 \beta$ from the monocytes.

\section{Association between innate cytokines under different conditions of micronutrients and malaria status}

The association between IL-1 $\beta$ and IL-10 was found to be influenced by zinc status (Figure 4). The two innate cytokines TNF and IL-1 $\beta$ are a prerequisite in early responses to malaria infection and IL-10 is an important regulatory cytokine affected by nutrient deficiencies and malaria infection status. This is critical under tropical situations where both micronutrients deficiencies and malaria prevail, posing a challenge to the early immune response to infections.

\section{Conclusions}

In conclusion, it was shown micronutrient deficiencies to variably influence some in vitro innate cytokine concentrations. Zinc deficiency in particular, was found to possibly influence the in vitro production of various innate cytokines that particularly are modulated by malaria status. Magnesium deficiency, on the other hand, seemed to associate with higher concentrations of IL-10 in donors uninfected at time of blood collection. These results may be speculative indicators that while zinc deficiency and possibly iron deficiency anaemia might increase proinflammatory cytokines such as IL- $1 \beta$ and TNF, magnesium deficiency may have greater influence on antiinflammatory cytokines such as IL-10. With regards to early innate cytokine responses to malaria, an ideal situation should be to supplement children with a combination of a few precisely selected micronutrients rather than single nutrients, although further studies involving larger sample sizes still need to be performed. This study has indicated the effect of poor nutrition on innate immune responses in children from malaria endemic area and how malaria infection may modulate these relationships. The findings have also shown plasticity in cytokine profiles of mononuclear cells reacting to malaria infection under conditions of different micronutrient deficiencies. Our findings therefore lay the foundations for future inclusion of selected micronutrients in malaria vaccine intervention programmes, particularly in developing countries, to boost immune response to malaria.

\section{Conflict of interests}

The authors declare that they have no competing interests.

\section{Authors' contributions}

EM carried out the protocol development and the laboratory analysis and drafted the manuscript. MM participated in cell culture protocol development, and laboratory analysis. JV: conducted the inclusion of the patients and the field work. MMcC: assisted in interpretation of data. JS and RO co-directed the field work; HV: conceived the study and acquired the funds, performed the statistical analysis and assisted in manuscript preparation. HS directed the study, supervised protocol development and manuscript preparation. All authors read and approved the final manuscript.

\section{Acknowledgements}

We received financial support from the Netherlands Organization for Scientific Research, NWO/WOTRO (grant numbers W93-413, WAO93-441, and WIZ93465) and UN Children's Fund (UNICEF). HV is currently supported by the European Community's Seventh Framework Programme under grant agreement no 211484 . We gratefully acknowledge the participating children, parents and the assistance given by community leaders, volunteers and colleagues in Tanzania and The Netherlands, and Marga van de Vegte, Henry Witteveen and Robert Sauerwein, Radboud University, Nijmegen, The Netherlands, for providing parasitized erythrocytes. We thank Dr. Jos PM Wielders, Meander Medical Centre Amersfoort, The Netherlands for measurements of micronutrients. This paper was published with support of the Executive Director of KCMC, Moshi and the Director-General of the National Institute for Medical Research, Dar es Salaam, Tanzania. Marita Troye-Blomberg and Nele Wellinghausen are acknowledged for their material support.

\section{Author Details}

${ }^{1}$ Cell Biology and Immunology Group, Wageningen University, The Netherlands, ${ }^{2}$ Host-Microbe Interactomics, Wageningen University, The Netherlands, ${ }^{3}$ Danone Research, Wageningen, The Netherlands, ${ }^{4}$ Department of Medical Microbiology, Radboud University, Nijmegen, The Netherlands, ${ }_{5}^{5}$ Kilimanjaro Christian Medical Centre (KCMC), Moshi, Tanzania, ${ }^{6} L o n d o n$ School of Hygiene and Tropical Medicine, Nutrition and Public Health Intervention Research Unit, London, UK and 7Muhimbili University of Health and Allied Sciences, Biochemistry Department, School of Medicine, Dar es Salaam, Tanzania

Received: 21 December 2009 Accepted: 17 May 2010 Published: 17 May 2010

\section{References}

1. Rink L, Haase H: Zinc homeostasis and immunity. Trends Immuno/ 2007, 28:1-4.

2. Wellinghausen $N$, Kirchner $H$, Rink L: The immunobiology of zinc. Immunol Today 1997, 11:519-521.

3. Wieringa FT, Dijkhuizen MA, West CE, Ven-Jongekrijg J van der, Muhilal, Meer JWM van der: Reduced production of immunoregulatory cytokines in vitamin A- and zinc-deficient Indonesian infants. Eur J Clin Nutr 2004, 58:1498-1504

4. Black RE: Therapeutic and preventive effects of zinc on serious childhood infectious diseases in developing countries. Am J Clin Nutr $1998,68: 4765-4795$

5. Muller $\mathrm{O}$, Becher $H$, van Zweeden AB, Ye Y, Diallo DA, Konate AT, Gbangou A, Kouyate B, Garenne M: Effect of zinc supplementation on malaria and other causes of morbidity in west African children: randomised double blind placebo controlled trial. BMJ 2001, 322:1567.

6. Shankar AH, Genton B, Baisor M, Paino J, Tamja S, Adiguma T, Wu L, Rare L, Bannon D, Tielsch JM, Alpers MP, West KP Jr: The influence of zinc supplementation on morbidity due to Plasmodium falciparum: a randomized trial in preschool children in Papua New Guinea. Am J Trop Med Hyg 2000, 62:663-669.

7. Shankar AH, Prasad AS: Zinc and immune function: the biological basis of altered resistance to infection. Am J Clin Nutr 1998, 68:447S-463S.

8. Percival SS: Neutropenia caused by copper deficiency: possible mechanisms of action. Nutr Rev 1995, 53:59-66.

9. Erickson KL, Medina EA, Hubbard NE: Micronutrients and Innate Immunity. J Infect Dis 2000, 182:S5-S10.

10. Walther M, Woodruff J, Edele F, Jeffries D, Tongren JE, King E, Andrews L, Bejon P, Gilbert SC, De Souza JB, Sinden R, Hill AV, Riley EM: Innate immune responses to human malaria: heterogeneous cytokine 
responses to blood-stage Plasmodium falciparum correlate with parasitological and clinical outcomes. J Immuno/ 2006, 177:5736-5745.

11. Keen CL, Gershwin ME: Zinc deficiency and immune function. Annu Rev Nutr 1990, 10:415-431.

12. Walker CF, Black RE: Zinc and the risk for infectious disease. Ann Rev Nutr 2004, 24:255-275.

13. Ibs K-H, Rink L: Zinc-Altered Immune Function. J Nutr 2003 , 133:1452S-1456S.

14. Rink L, Gabriel P: Extracellular and immunological actions of zinc. BioMetals 2001, 14:367-383.

15. McKay I, Rosen FS: Innate immunity. N Engl J Med 2000, 343:338-344

16. Stevenson MM, Riley EM: Innate immunity to malaria. Nat Rev Immunol 2004, 4:169-180.

17. Pallauf J, Rimbach G: Nutritional significance of phytic acid and phytase. Arch Animal Nutr 1997, 50:301-319.

18. Veenemans J, Andang'o PEA, Mbugi EV, Kraaijenhagen RJ, Mwaniki DL, Mockenhaupt FP, Roewer S, Olomi RM, Shao JF, Meer JWM van der, Savelkoul HFJ, Verhoef $\mathrm{H}$ : $\mathbf{a}^{+}-$Thalassemia protects against anemia associated with asymptomatic malaria: evidence from communitybased surveys in Tanzania and Kenya. J Infect Dis 2008, 198:401-408.

19. Moody A: Rapid diagnostic tests for malaria parasites. Clin Microbiol Rev 2002, 15:66-78.

20. Piper R, Lebras J, Wentworth L, Hunt-Cooke A, Houzé S, Chiodini P, Makler $\mathrm{M}$ : Immunocapture diagnostic assays for malaria using Plasmodium lactate dehydrogenase (pLDH). Am J Trop Med Hyg 1999, 60:109-18.

21. Ryan A: Analysis of blood serum on the Liberty Series II ICP-AES with the axially-viewed plasma. ICP-24: Varian Inc 1998:1-9.

22. Jeurink PV, Vissers YM, Rappard B, Savelkoul HFJ: T cell responses in fresh and cryopreserved peripheral blood mononuclear cells: Kinetics of cell viability, cellular subsets, proliferation, and cytokine production. Cryobiol 2008, 57:91-103.

23. Hartgers FC, Obeng BB, Kruize YCM, Dijkhuis A, McCall M, Sauerwein RW Luty AJF, Boakye DA, Yazdanbakhsh M: Responses to malarial antigens are altered in helminth-infected children. J Inf Dis 2009, 199:1528-1535.

24. McCall MBB, Netea MG, Hermsen CC, Jansen T, Jacobs L, Golenbock D, Ven AJAM van der, Sauerwein RW: Plasmodium falciparum infection causes proinflammatory priming of human TLR responses. J Immunol 2007, 179:162-171.

25. Rivadeneira EM, Wasserman M, Espinal CT: Separation and concentration of schizonts of Plasmodium falciparum by Percoll gradients. J Eukary Microbiol 1983, 30:367-370.

26. Artavanis-Tsakonas K, Riley EM: Innate immune response to malaria: rapid induction of IFN- $\gamma$ from human NK cells by live Plasmodium falciparum-infected erythrocytes. J Immunol 2002, 169:2956-2963.

27. Yssel H, De Vries JE, Koken M, Van Blitterswijk W, Spits H: Serum-free medium for generation and propagation of functional human cytotoxic and helper T cell clones. J Immunol Methods 1984, 72:219-227.

28. Imada M, Simons FE, Jay FT, HayGlass KT: Antigen mediated and polyclonal stimulation of human cytokine production elicit qualitatively different patterns of cytokine gene expression. Int Immunol 1995, 7:229-237.

29. Lichodziejewska MDB, Kos MDJ, Rezler MDJ, Grudzka MDK, Duzzniewska MDM, Budaj MDA, Ceremuzynski MDPL: Clinical symptoms of mitral valve prolapse are related to hypomagnesemia and attenuated by magnesium supplementation. Am J Cardiol 1997, 79:768-772.

30. Naderi ASA, Reilly RF: Hereditary etiologies of hypomagnesemia. Nat Clin Pract Neph 2008, 4:80-89.

31. Perkins DJ, Weinberg JB, Kremsner PG: Reduced interleukin-12 and Transforming Growth Factor-beta in severe childhood malaria: relationship of cytokine balance with disease severity. J Infect Dis 2000, 182:988-992.

32. Ramharter M, Willheim M, Winkler H, Wahl K, Lagler H, Graninger W, Winkler S: Cytokine profile of Plasmodium falciparum-specific T cells in non-immune malaria patients. Parasite Immunol 2003, 25:211-219.

33. Wintergerst ES, Maggini S, Hornig DH: Contribution of selected vitamins and trace elements to immune function. Ann Nutr Metab 2007, 51:301-323.

34. Artavanis-Tsakonas K, Tongren JE, Riley EM: The war between the malaria parasite and the immune system: immunity, immunoregulation and immunopathology. Clin Exp Immunol 2003, 133:145-152

35. Prasad A: Zinc: mechanisms of host defense. J Nutrition 2007 , 137:1345-1349
36. Loharungsikul S, Troye-Blomberg M, Amoudruz P, Pichyangkul S, Yongvanitchit K, Looareesuwan S, Mahakunkijcharoen Y, Sarntivijai S, Khusmith S: Expression of Toll-like receptors on antigen-presenting cells in patients with falciparum malaria. Acta Trop 2008, 105:10-15.

37. Krishnegowda G, Hajjar AM, Zhu J, Douglass EJ, Uematsu S, Akira S, Woods AS, Gowda DC: Induction of proinflammatory responses in macrophages by the glycosylphosphatidylinositols of Plasmodium falciparum: cell signaling receptors, glycosylphosphatidylinositol (GPI) structural requirement, and regulation of GPI activity. J Biol Chem 2005, 280:8606-8616

38. Parroche $P$, Lauw FN, Goutagny N, Latz E, Monks BG, Visintin A, Halmen KA, Lamphier M, Olivier M, Bartholomeu DC, Gazzinelli RT, Golenbock DT: Malaria hemozoin is immunologically inert but radically enhances innate responses by presenting malaria DNA to Toll-like receptor 9. Proc Nat Acad Sci USA 2007, 104:1919-1924

39. Hartgers FC, Obeng BB, Voskamp A, Larbi IA, Amoah AS, Luty AJF, Boakye D, Yazdanbakhsh M: Enhanced Toll-Like Receptor responsiveness associated with mitogen-activated protein kinase activation in Plasmodium falciparum-infected children. Infect Immun 2008, 76:5149-5157.

40. Rivera MT, De Souza AP, Araujo-Jorge TC, De Castro SL, Vanderpas J: Trace elements, innate immune response and parasites. Clin Chem Lab Med 2003, 41:1020-1025.

41. Overbeck S, Rink L, Haase H: Modulating the immune response by oral zinc supplementation: a single approach for multiple diseases. Arch Immunol Ther Exp 2008, 56:15-30.

42. Haase H, Rink L: Signal transduction in monocytes: the role of zinc ions. BioMetals 2007, 20:579-585.

43. Prasad AS, Bao B, Beck FWJ, Kucuk O, Sarkar FH: Antioxidant effect of zinc in humans. Free Radic Biol Med 2004, 37:1182-1190

44. Wellinghausen N, Martin M, Rink L: Zinc inhibits interleukin-1dependent T cell stimulation. Eur J Immunol 1997, 27:2529-2535.

45. von Bulow $\bigvee$, Dubben $S$, Engelhardt G, Hebel S, Plumakers B, Heine H, Rink $L$, Haase $H$ : Zinc-dependent suppression of TNF-alpha production is mediated by protein kinase A-induced inhibition of Raf-1, I kappa B kinase beta, and NF-kappa B. J Immunol 2007, 179:4180-4186.

46. von Bulow V, Rink L, Haase H: Zinc-mediated inhibition of cyclic nucleotide phosphodiesterase activity and expression suppresses TNF-alpha and IL-1 beta production in monocytes by elevation of guanosine 3',5'-cyclic monophosphate. J Immunol 2005, 175:4697-4705.

47. Keller CC, Yamo O, Ouma C, Ong'echa JM, Ounah D, Hittner JB, Vulule JM, Perkins DJ: Acquisition of hemozoin by monocytes down-regulates interleukin-12 p40 (IL-12p40) transcripts and circulating IL-12p70 through an IL-10-dependent mechanism: in vivo and in vitro findings in severe malarial anemia. Infect Immun 2006, 74:5249-5260.

48. Uh HW, Hartgers FC, Yazdanbakhsh M, Houwing-Duistermaat JJ: Evaluation of regression methods when immunological measurements are constrained by detection limits. BMC Immuno/ 2008, 9:59.

49. Scragg IG, Hensmann M, Bate CAW, Kwiatkowski D: Early cytokine induction by Plasmodium falciparumis not a classical endotoxin-like process. Eur J Immunol 1999, 29:2636-2644.

50. Prasad AS: Effects of zinc deficiency on Th1 and Th2 cytokine shifts. $J$ Inf Dis 2000, 182:S62-68.

51. Omer FM, Kurtzhals JAL, Riley EM: Maintaining the immunological balance in parasitic infections: a role for TGF- $\beta$ ? Parasitol Today 2000 16:18-23.

52. Torre D, Speranza F, Martegani R: Role of proinflammatory and antiinflammatory cytokines in the immune response to Plasmodium falciparum malaria. Lancet Infect Dis 2002, 2:719-720.

53. Clark IA, Rockett KA: The cytokine theory of human cerebral malaria. Parasitol Today 1994, 10:410-412.

54. Perlmann $\mathrm{P}$, Troye-Blomberg M: Malaria and the immune system in humans. Chem Immunol 2002, 80:229-242.

doi: 10.1186/1475-2875-9-130

Cite this article as: Mbugi et al., Alterations in early cytokine-mediated immune responses to Plasmodium falciparum infection in Tanzanian children with mineral element deficiencies: a cross-sectional survey Malaria Journal 2010, 9:130 